\title{
A nonlinear equation for fluids in multiconnected domain
}

\author{
Marin Marin', Ravi P Agarwal ${ }^{2,3}$ and Olivia A Florea1*
}

"Correspondence:

olivia.florea@unitbv.ro

${ }^{1}$ Faculty of Mathematics and

Computer Science, Transilvania

University of Brasov, Iuliu Maniu Str.,

No. 50, Brasov, Romania

Full list of author information is

available at the end of the article

\begin{abstract}
In this paper we propose a mathematical model for Reynolds' equation of a compressible fluid on a multiconnected field which simulates the function of a hybrid bearing. The boundary conditions on the inner boundaries are derived from the flow-rate continuity through the supplying orifices and are expressed by means of an integro-differential nonlinear equation. We propose a numerical method to solve this mathematical model.
\end{abstract}

Keywords: bearing; Reynolds' nonlinear equation; multiconnected field; 'column-wise' technique

\section{Introduction}

There are many studies dedicated to hybrid bearings. Some of such papers can be found in the complete book [1]. The aim of our present paper is to generalize the studies (done, for instance, in [2-4]) of hybrid bearings. In the previous papers, the domain in which the equation of Reynolds was solved is supposed multiconnected field with inside boundaries represented by continuous lines, so-called 'supplying lines.'

We consider a multiconnected domain in which the inside frontiers have a rectangular form, which has permitted, through passing to the limit of the dimensions and of the number of frontiers, the simulation of a supplying line, so we could compare our results with those of above mentioned researchers. The physical situation considered in this paper has the feature that the boundary conditions on the inner boundaries are of more difficult forms. These boundary conditions become nonlinear integral-differential expressions with respect to the solution of the Reynolds equation. With the aim to compare our results with those reported in the open literature, we consider that the inner boundaries are disposed in a normal plane to the bearing surfaces, but it is worth to mention that this is not a mandatory condition for our method. An iterative procedure of linearization, as proposed in [5-7], is used in the present study. The resulting algebraic system is solved on using a 'column-wise' technique (see, for instance, Castelli and Pirvies [2]).

In [8] the authors use the Cesaro means for the components of energy and prove the asymptotic equipartition in mean of the kinetic and strain energies. Some results from the theory of semigroups of linear operators are used in [9] in order to prove the existence and uniqueness of a weak solution. The paper [10] is dedicated to heat conduction in deformable bodies which depends on two temperatures. The first one is the conductive

(c) 2015 Marin et al. This article is distributed under the terms of the Creative Commons Attribution 4.0 International License (http://creativecommons.org/licenses/by/4.0/), which permits unrestricted use, distribution, and reproduction in any medium, provided you give appropriate credit to the original author(s) and the source, provide a link to the Creative Commons license, and indicate if changes were made. 
temperature, the second is the thermodynamic temperature, and the difference between them is proportional to the heat supply.

Also, we must outline the minimum principle in [11] and remark that the solution proposed in [12] can be used for Maxwell's fluids and, also, for Newtonian fluids.

\section{Basic notions}

Consider a circular bearing supplying with a compressible fluid through feed pocket disposed in planes normal on the bearing axis. The geometrical elements which appear in our mathematical model, are:

- $L=$ the length of the bearing;

$-R=$ the radius of the shaft;

- $C=$ the radial clearance;

$-\varepsilon=$ the eccentricity;

- $c=$ the length of a feed pocket;

$-d=$ the width of a feed pocket;

- $\theta=$ the polar angle measured from the line of centers bearing surfaces;

- $H=$ the thickness of fluid film, $H=C+\varepsilon \cos \theta$.

We use a Cartesian system of orthogonal axes such that the $O x_{1}$ and $O x_{2}$ are included in the bearing shaft surface, with the origin $O$ in the intersection of the normal plane on the rotation axis, in an end of the bearing, with the plane determined by the axes of bearing shaft and bearing baking. Also, the $O x_{3}$-axis is parallel with the rotation axis, the $O x_{1}$ has the sense of the rotation of bearing shaft. In this system the coordinates of the centers of the feed pockets are $\left(x_{1 m}, x_{3 m}\right)$, where $m$ is a generic index. If the number of feed pockets is $n_{0}$, then $m=\overline{1, n_{0}}$. The domain represented by the surface of the bearing shaft, on which are projected the feed pockets can by assimilated with a domain of band type, having width $L$ in the direction of $O x_{3}$ and being periodic in the direction of $O x_{1}$, with period $2 \pi R$. Based on the periodicity, we can consider the mathematical model in the domain

$$
\mathcal{D}=[0,2 \pi] \times[0, L] \backslash \bigcup_{m} D_{m}
$$

where

$$
D_{m}=\left(x_{1 m}-\frac{c}{2}, x_{1 m}+\frac{c}{2}\right) \times\left(x_{3 m}-\frac{d}{2}, x_{3 m}+\frac{d}{2}\right),
$$

with $c$ the length of a feed pocket and $d$ the width of a feed pocket.

\section{The mathematical model}

We use the notations:

- $P=$ the pressure in the fluid film;

- $H=$ the gap between the rotating shaft and the bushing;

- $\varrho=$ the density of the fluid;

- $\mu=$ the dynamic viscosity of the fluid;

- $V=$ the relative speed of the axis bearing;

- $p_{a}=$ the pressure on the ends of the bearing.

With these notations, the mathematical model becomes [11]: 
Figure 1 Journal bearing schematic.

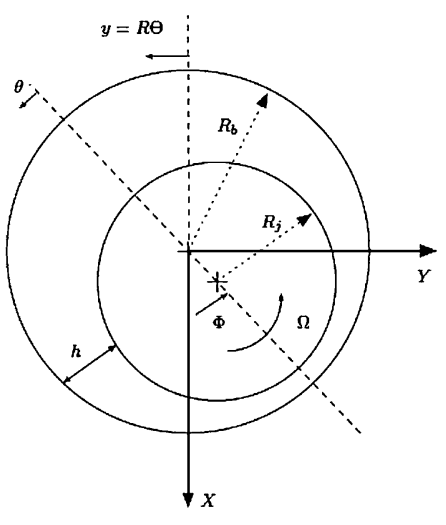

Figure 2 Definition of the journal eccentricity.

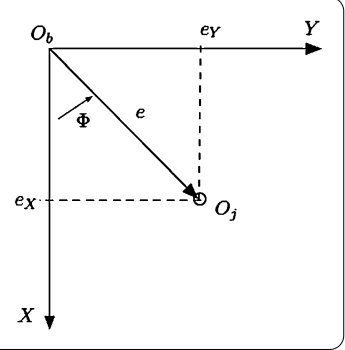

- the Reynolds equation for the steady state condition of a gas journal bearing:

$$
\frac{\partial}{\partial x_{1}}\left(P H^{3} \frac{\partial P}{\partial x_{1}}\right)+\frac{\partial}{\partial x_{3}}\left(P H^{3} \frac{\partial P}{\partial x_{3}}\right)=6 \mu V \frac{\partial}{\partial x_{1}}(P H) ;
$$

- the equation of state, which is supplemented with the assumption that the gas undergoes an isothermal process:

$$
\varrho=\frac{P}{\mathcal{R} T}
$$

- the conditions on boundary of the domain $\mathcal{D}$, where (1) is applied:

$$
\begin{aligned}
& P\left(x_{1}, 0\right)=P\left(x_{1}, L\right)=p_{a} \\
& \oint_{C_{m}} \varrho\left(\frac{\mathbf{V} H}{2}-\frac{H^{3}}{12 \mu} \operatorname{grad} P\right) \mathbf{n} d l=q_{m}, \quad\left(x_{1}, x_{3}\right) \in C_{m},
\end{aligned}
$$

where the integral represents the flow-rate through the surface into the surface determined by $C_{m}$, the boundary of the domain $D_{m}$, and the generators of length $H$. $q_{m}$ is the flow-rate through the incoming orifice ( $\mathbf{n}$ is the outside normal of the curve $\left.C_{m}\right)$.

The location of the minimum film thickness is defined by the attitude angle $\Phi$ (see Figures 1 and 2). The film thickness function $h=h\left(x_{3}, x_{1}\right)$ depends on the position of the center of the shaft according to

$$
h=C+e_{x} \cos (\Theta)+e_{y} \sin (\Theta)
$$

where $C=R_{b}-R_{j}$ is the radial clearance of the bearing and $\Theta=\theta+\Phi$. 
In the equations of the mathematical model, we use the following non-dimensional variables:

$$
\theta=\frac{x_{1}}{R}, \quad h=\frac{H}{C}=1+e \cos \theta, \quad \omega=\frac{V}{R}, \quad z=\frac{x_{3}}{R}, \quad p=\frac{P}{p_{a}} .
$$

With these variables, (1) and the conditions (2) and (3) become

$$
\begin{aligned}
& \frac{\partial}{\partial \theta}\left(p h^{3} \frac{\partial p}{\partial \theta}\right)+\frac{\partial}{\partial z}\left(p h^{3} \frac{\partial p}{\partial z}\right)=\Lambda \frac{\partial}{\partial \theta}(p h), \\
& p(\theta, 0)=p\left(\theta, \frac{L}{R}\right)=1 \\
& \oint_{C_{m}}\left(\Lambda p \mathbf{i}_{1}-R h^{3} p \operatorname{grad} P\right) \mathbf{n} d \mathbf{l}=\mathbf{q}_{m}
\end{aligned}
$$

where

$$
\begin{aligned}
& \Lambda=\frac{6 \mu \omega}{p_{a}}\left(\frac{R}{C}\right)^{2} \text { is the bearing number, } \\
& q_{m}=\frac{2 \pi \Lambda_{s}}{n_{0}}\left(\frac{p_{s}}{p_{a}}\right)^{2} \sqrt{\frac{1+\delta^{2}}{h_{m}^{2}+\delta^{2}}} h_{m} G_{m}, \\
& \delta=\frac{a^{2}}{d C} \\
& \Lambda_{s}=\frac{6 \mu n_{0} a^{2}}{p_{s} C^{3}} \sqrt{\frac{\mathcal{R} T}{1+\delta^{2}}}, \\
& G_{m}=C_{D} \gamma^{2}\left(\frac{2}{\gamma+1}\right)^{(\gamma+1) / 2(\gamma-1)}, \quad \text { if } \frac{p_{m}}{p_{s}} \leq\left(\frac{2}{\gamma+1}\right)^{\gamma /(\gamma-1)}, \\
& G_{m}=C_{D}\left\{\frac{2 \gamma}{\gamma-1}\left(\frac{p_{m}}{p_{s}}\right)^{\frac{2}{\gamma}}\left[1-\left(\frac{p_{m}}{p_{s}}\right)^{\frac{\gamma-1}{\gamma}}\right]\right\}^{\frac{1}{2}}, \quad \text { if }\left(\frac{2}{\gamma+1}\right)^{\frac{\gamma}{\gamma-1}}<\frac{p_{m}}{p_{s}} \leq 1 ;
\end{aligned}
$$

where $\theta$ and $z$ are the coordinates in the circumferential and axial direction, respectively; $c$ is the radial clearance, $h$ is the thickness of the fluid film on the ridge region;

- $p_{s}=$ the supplying pressure;

- $p_{m}=$ the pressure on the curve $C_{m}$;

- $h_{m}=$ the film thickness on the curve $C_{m}$;

- $a=$ the radius of the feed orifice;

- $\gamma=$ the adiabatic constant and its value is about 1,405 ;

- $C_{D}=$ a coefficient, depending on the form of the feed orifice, experimentally was obtained: $C_{D} \in[0.6,1]$.

Because of the fact that the coefficients of (5) depend only on the parameter $\Lambda$, which is constant with regard to $\theta$ and $z$, and with regard to $h$, which is a periodical function on $\theta$, one finds, as a result, the solution of the equation is periodical:

$$
p(\theta, z)=p(\theta+2 \pi, z)
$$

It is easy to see that the equations of the mathematical model are nonlinear. The linearization of these equations is made, in the usual way, by introducing the function $Q=(p h)^{2}$. 
Figure 3 The representation of the curve $C_{m}$.

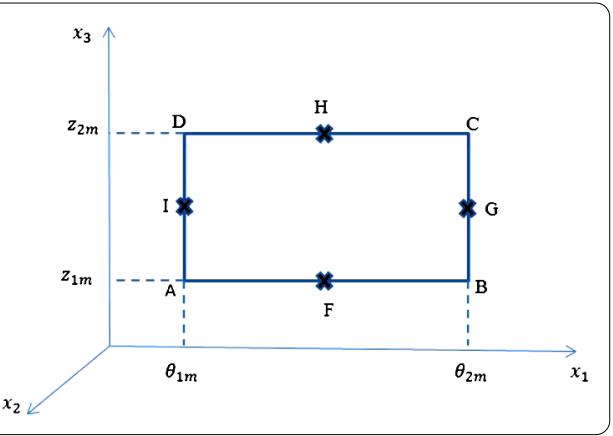

With this new variable, (5), (6), and (9) become

$$
\begin{aligned}
& \frac{\partial^{2} Q}{\partial \theta^{2}}+\frac{\partial^{2} Q}{\partial z^{2}}-\frac{1}{h}\left(\frac{\partial h}{\partial \theta}+\frac{\Lambda}{p h}\right) \frac{\partial Q}{\partial \theta}-\frac{2}{h} \frac{\partial^{2} h}{\partial \theta^{2}} Q=0, \\
& Q(\theta, 0)=Q\left(\theta, \frac{L}{R}\right)=h^{2}, \\
& Q(\theta, z)=Q(\theta+2 \pi, z) .
\end{aligned}
$$

In order to obtain the new form of (7), we consider the domain corresponding to a feed pocket.

Suppose that the pressure is constant on the boundary of the feed pocket,

$$
p(\theta, z)=p_{m}, \quad(\theta, z) \in C_{m}
$$

and consider a mean value of $h$ on $C_{m}$, that is, $\tilde{h}_{m}$, and a mean value of $q_{m}$ on $C_{m}$, that is, $\tilde{q}_{m}$. Then we have as a result a constant value for $Q$ :

$$
Q(\theta, z)=\left(p_{m} \tilde{h}_{m}\right)^{2}=Q_{m}, \quad(\theta, z) \in C_{m}
$$

The outline $C_{m}$ is a rectangle with the sides $A B\|C D\| O x_{1}$ and $B C\|D A\| O x_{3}$ (see Figure 3). The equations of the sides are:

- $A B: z=z_{1 m}, \theta \in\left[\theta_{1 m}, \theta_{2 m}\right]$;

- $B C: \theta=\theta_{2 m}, z \in\left[z_{1 m}, z_{2 m}\right]$;

- CD: $z=z_{2 m}, \theta \in\left[\theta_{1 m}, \theta_{2 m}\right]$;

- DA: $\theta=\theta_{1 m}, z \in\left[z_{1 m}, z_{2 m}\right]$.

With these hypotheses, (7) becomes

$$
\begin{aligned}
\mathbf{q}_{m}= & \oint_{C_{m}}\left(\Lambda p h \mathbf{i}_{1}-R h^{3} p \operatorname{grad} P\right) \mathbf{n} d \mathbf{l} \\
= & \int_{A B}\left[\Lambda p h \mathbf{i}_{1}-h^{3} p\left(\frac{\partial p}{\partial \theta} \mathbf{i}_{1}+\frac{\partial p}{\partial z} \mathbf{i}_{3}\right)\right] \mathbf{n}_{A B} d \mathbf{l} \\
& +\int_{B C}\left[\Lambda p h \mathbf{i}_{1}-h^{3} p\left(\frac{\partial p}{\partial \theta} \mathbf{i}_{1}+\frac{\partial p}{\partial z} \mathbf{i}_{3}\right)\right] \mathbf{n}_{B C} d \mathbf{l} \\
& +\int_{C D}\left[\Lambda p h \mathbf{i}_{1}-h^{3} p\left(\frac{\partial p}{\partial \theta} \mathbf{i}_{1}+\frac{\partial p}{\partial z} \mathbf{i}_{3}\right)\right] \mathbf{n}_{C D} d \mathbf{l}
\end{aligned}
$$




$$
\begin{aligned}
& +\int_{D A}\left[\Lambda p h \mathbf{i}_{1}-h^{3} p\left(\frac{\partial p}{\partial \theta} \mathbf{i}_{1}+\frac{\partial p}{\partial z} \mathbf{i}_{3}\right)\right] \mathbf{n}_{D A} d \mathbf{l} \\
= & \int_{A B} h^{3} p \frac{\partial p}{\partial z} d \theta+\int_{B C}\left(\Lambda p h-h^{3} p \frac{\partial p}{\partial \theta}\right) d z \\
& +\int_{C D} h^{3} p \frac{\partial p}{\partial z} d \theta+\int_{D A}\left(\Lambda p h-h^{3} p \frac{\partial p}{\partial \theta}\right) d z, \\
\tilde{q}_{m}= & \frac{1}{2 \tilde{h}_{m}^{2}}\left[\int_{A B} h^{3} \frac{\partial Q}{\partial z} d \theta+\frac{2 \Lambda h_{G}}{p_{m}}\left(z_{C}-z_{B}\right) Q_{m}-h_{G}^{3} \int_{B C} \frac{\partial Q}{\partial \theta} d z\right. \\
& \left.+\int_{C D} h^{3} \frac{\partial Q}{\partial \theta} d \theta+\frac{2 \Lambda h_{1}}{p_{m}}\left(z_{A}-z_{D}\right) Q_{m}-h_{1}^{3} \int_{D A} \frac{\partial Q}{\partial \theta} d z\right] .
\end{aligned}
$$

We approximate the derivatives of the function $Q$ on the outline by the values in the points $F, G, H, I$ (the mid-points of the sides $A B, B C, C D, D A$ ):

$$
\begin{array}{ll}
\left(\frac{\partial Q}{\partial z}\right)_{A B} \approx\left(\frac{\partial Q}{\partial z}\right)_{F}, \quad\left(\frac{\partial Q}{\partial \theta}\right)_{B C} \approx\left(\frac{\partial Q}{\partial \theta}\right)_{G}, \\
\left(\frac{\partial Q}{\partial z}\right)_{C D} \approx\left(\frac{\partial Q}{\partial z}\right)_{H}, \quad\left(\frac{\partial Q}{\partial \theta}\right)_{D A} \approx\left(\frac{\partial Q}{\partial z}\right)_{I},
\end{array}
$$

such that, for the flow, we obtain the expression

$$
\begin{aligned}
\tilde{q}_{m}= & \frac{1}{2 \tilde{h}_{m}^{2}}\left\{\left[\left(\frac{\partial Q}{\partial z}\right)_{F}-\left(\frac{\partial Q}{\partial z}\right)_{H}\right] \int_{A B} h^{3} d \theta\right. \\
& \left.-\overline{B C}\left[h_{G}^{3}\left(\frac{\partial Q}{\partial \theta}\right)_{G}-h_{1}\left(\frac{\partial Q}{\partial \theta}\right)_{I}\right]+\frac{2 \Lambda \overline{B C}}{p_{m}}\left(h_{G}-h_{1}\right) Q_{m}\right\} .
\end{aligned}
$$

To obtain the convergence of our procedure, the function $G_{m}$ from (8) is prolonged on the domain $p_{m} / p_{s}>1$.

\section{The solution}

In order to solve the system of equations of the mathematical model, we use the method of finite difference. The domain $\mathcal{D}$ is covered with a grid of parallel lines with $O x_{1}$ and $O x_{3}$. The feed pocket center is a node. We have

$$
\begin{aligned}
& G_{m}=C_{D} \gamma^{1 / 2}\left(\frac{2}{\gamma+1}\right)^{(\gamma+1) / 2(\gamma-1)}, \quad \text { if } \frac{p_{m}}{p_{s}} \leq\left(\frac{2}{\gamma+1}\right)^{\gamma /(\gamma-1)}, \\
& G_{m}=C_{D}\left\{\frac{2 \gamma}{\gamma-1}\left(\frac{p_{m}}{p_{s}}\right)^{\frac{2}{\gamma}}\left[1-\left(\frac{p_{m}}{p_{s}}\right)^{\frac{\gamma-1}{\gamma}}\right]\right\}^{\frac{1}{2}}, \quad \text { if }\left(\frac{2}{\gamma+1}\right)^{\frac{\gamma}{\gamma-1}}<\frac{p_{m}}{p_{s}} \leq 1, \\
& G_{m}=-C_{D}\left\{\frac{2 \gamma}{\gamma-1}\left(\frac{p_{m}}{p_{s}}\right)^{\frac{2}{\gamma}}\left[1-\left(\frac{p_{m}}{p_{s}}\right)^{\frac{\gamma-1}{\gamma}}\right]\right\}^{\frac{1}{2}}, \quad \text { if } \frac{p_{m}}{p_{s}}>1 .
\end{aligned}
$$

If the dimensions of the grid are $N \times M$, then the coordinates of a node are

$$
\begin{array}{ll}
\theta_{j}=(j-1) \Delta \theta, & j=\overline{1, N}, \\
z_{i}=(i-1) \Delta z, & i=\overline{1, M},
\end{array}
$$


where

$$
\Delta \theta=\frac{2 \pi}{N-1}, \quad \Delta z=\frac{L}{R(M-1)} .
$$

If we use the notations $Q\left(\theta_{j}, z_{i}\right)=Q_{j, i}$, then the derivatives can be written as follows:

$$
\begin{aligned}
& \left(\frac{\partial Q}{\partial \theta}\right)_{j, i}=\frac{Q_{j+1, i}-Q_{j-1, i}}{2 \Delta \theta}, \quad\left(\frac{\partial Q}{\partial z}\right)_{j, i}=\frac{Q_{j, i+1}-Q_{j, i-1}}{2 \Delta z}, \\
& \left(\frac{\partial^{2} Q}{\partial \theta^{2}}\right)_{j, i}=\frac{Q_{j+1, i}-2 Q_{j, i}+Q_{j-1, i}}{(\Delta \theta)^{2}}, \quad\left(\frac{\partial^{2} Q}{\partial z^{2}}\right)_{j, i}=\frac{Q_{j, i+1}-2 Q_{j, i}+Q_{j, i-1}}{(\Delta z)^{2}} .
\end{aligned}
$$

In the neighborhood of the feed pocket, the formulas will be modified.

The derivatives of the functions $Q$, in the node $(k, l)$ in the neighborhood of the outline $C_{m}$ can be calculated as follows:

- if $(l-1) \cdot \Delta z \in\left[z_{1 m}, z_{2 m}\right]$ and $0<k \cdot \Delta \theta-\theta_{m 1}=\Delta \theta_{1}<\Delta \theta$, then

$$
\begin{aligned}
& \left(\frac{\partial Q}{\partial \theta}\right)_{k, l}=\frac{\Delta \theta}{\Delta \theta_{1}\left(\Delta \theta+\Delta \theta_{1}\right)} Q_{m}-\frac{\Delta \theta-\Delta \theta_{1}}{\Delta \theta \Delta \theta_{1}} Q_{k, l}-\frac{\Delta \theta_{1}}{\Delta \theta_{1}\left(\Delta \theta+\Delta \theta_{1}\right)} Q_{k-1, l} \\
& \left(\frac{\partial^{2} Q}{\partial \theta^{2}}\right)_{k, l}=\frac{2}{\Delta \theta_{1}\left(\Delta \theta+\Delta \theta_{1}\right)} Q_{m}-\frac{2}{\Delta \theta \Delta \theta_{1}} Q_{k, l}-\frac{2}{\Delta \theta_{1}\left(\Delta \theta+\Delta \theta_{1}\right)} Q_{k-1, l}
\end{aligned}
$$

- if $(l-1) \cdot \Delta z \in\left[z_{1 m}, z_{2 m}\right]$ and $0<(k-1) \cdot \Delta \theta-\theta_{m 2}<\Delta \theta$, then

$$
\begin{aligned}
& \left(\frac{\partial Q}{\partial \theta}\right)_{k, l}=\frac{\Delta \theta_{1}}{\Delta \theta_{1}\left(\Delta \theta+\Delta \theta_{1}\right)} Q_{k+1, l}+\frac{\Delta \theta-\Delta \theta_{1}}{\Delta \theta \Delta \theta_{1}} Q_{k, l}-\frac{\Delta \theta}{\Delta \theta_{1}\left(\Delta \theta+\Delta \theta_{1}\right)} Q_{m} \\
& \left(\frac{\partial^{2} Q}{\partial \theta^{2}}\right)_{k, l}=\frac{2}{\Delta \theta\left(\Delta \theta+\Delta \theta_{1}\right)} Q_{k+1, l}-\frac{2}{\Delta \theta \Delta \theta_{1}} Q_{k, l}+\frac{2}{\Delta \theta_{1}\left(\Delta \theta+\Delta \theta_{1}\right)} Q_{m} .
\end{aligned}
$$

With a similar procedure we can obtain the derivatives with regard to $z$ :

- if $(k-1) \cdot \Delta \theta \in\left[\theta_{m 1}, \theta_{m 2}\right]$ and $0<l \cdot \Delta z-z_{m 1}=\Delta z_{1}<\Delta z$, then

$$
\left(\frac{\partial Q}{\partial z}\right)_{k, l}=\frac{\Delta z}{\Delta z_{1}\left(\Delta z+\Delta z_{1}\right)} Q_{m}-\frac{\Delta z-\Delta z_{1}}{\Delta z \Delta z_{1}} Q_{k, l}-\frac{\Delta z_{1}}{\Delta z\left(\Delta z+\Delta z_{1}\right)} Q_{k, l-1}
$$

- if $(k-1) \cdot \Delta \theta \in\left[\theta_{m 1}, \theta_{m 2}\right]$ and $0<(l-1) \cdot \Delta z-z_{m 2}<\Delta z$, then

$$
\begin{aligned}
& \left(\frac{\partial Q}{\partial z}\right)_{k, l}=\frac{\Delta z_{1}}{\Delta z_{1}\left(\Delta z+\Delta z_{1}\right)} Q_{k+1, l}+\frac{\Delta z-\Delta z_{1}}{\Delta z \Delta z_{1}} Q_{k, l}-\frac{\Delta z}{\Delta z_{1}\left(\Delta z+\Delta z_{1}\right)} Q_{m}, \\
& \left(\frac{\partial^{2} Q}{\partial z^{2}}\right)_{k, l}=\frac{2}{\Delta z\left(\Delta z+\Delta z_{1}\right)} Q_{k+1, l}-\frac{2}{\Delta z \Delta z_{1}} Q_{k, l}+\frac{2}{\Delta z_{1}\left(\Delta z+\Delta z_{1}\right)} Q_{m} .
\end{aligned}
$$

In order to determine $Q_{m}$, we made in (13) the approximations

$$
\begin{array}{ll}
\left(\frac{\partial Q}{\partial z}\right)_{F}=\frac{Q_{m}-Q_{j, i_{1}}}{\Delta z_{1}}, & \left(\frac{\partial Q}{\partial z}\right)_{H}=\frac{Q_{j, i_{2}}-Q_{m}}{\Delta z_{1}}, \\
\left(\frac{\partial Q}{\partial \theta}\right)_{I}=\frac{Q_{m}-Q_{j_{1}, i}}{\Delta \theta_{1}}, & \left(\frac{\partial Q}{\partial \theta}\right)_{G}=\frac{Q_{j_{2}, i}-Q_{m}}{\Delta \theta_{1}},
\end{array}
$$


where

$$
\begin{array}{ll}
i_{1}=\frac{z_{m_{1}}-\Delta z_{1}}{\Delta z}+1, & i_{2}=\frac{z_{m_{2}}+\Delta z_{1}}{\Delta z}+1, \\
j_{1}=\frac{\theta_{m_{1}}-\Delta \theta_{1}}{\Delta \theta}+1, & j_{2}=\frac{\theta_{m_{2}}+\Delta \theta_{1}}{\Delta \theta}+1 .
\end{array}
$$

Substituting $h_{m}$ with $\tilde{h}_{m}$ in the expression of $\tilde{q}_{m}$ from (13), one finds, as a result,

$$
\begin{aligned}
\frac{4 \pi}{\Lambda_{s} p_{s} \tilde{h}_{m}^{3}} & \sqrt{\frac{1+\delta^{2}}{n_{0}}} G_{m} \\
= & {\left[\frac{2}{\Delta z_{1}} \int_{\theta_{m_{1}}}^{\theta_{m_{2}}} h^{3} d \theta+\frac{z_{m_{2}}-z_{m_{1}}}{\Delta \theta_{1}}\left(h_{G}^{3}+h_{1}^{3}\right)+\frac{2 \Lambda\left(z_{m_{2}}-z_{m_{1}}\right)}{p_{m}}\left(h_{G}-h_{1}\right)\right] Q_{m} } \\
& -\frac{1}{z P} \int_{\theta_{m_{1}}}^{\theta_{m_{2}}} h^{3} d \theta\left(Q_{j, i_{1}}+Q_{j, i_{2}}\right)-\frac{z_{m_{2}}-z_{m_{1}}}{\Delta \theta_{1}}\left(h_{G}^{3} Q_{j_{2}, i}+h_{1}^{3} Q_{j_{1}, i}\right) .
\end{aligned}
$$

As an initial iteration we use the function $Q^{(0)}$ which satisfies the boundary conditions (11) and the periodicity conditions (12), such that $Q^{(0)}=h^{2}$. In (21) we consider the unknown variable $x=p_{m} / p_{s}$. The coefficients of this variable can be calculated as a function of the previous iteration, such that (21) can be written in the form

$$
A G_{m}(x)-B^{(r-1)} x^{2}+C^{(r-1)}=0
$$

where

$$
\begin{aligned}
A= & \frac{4 \pi \Lambda_{s} p_{s}^{2} \tilde{h}_{m}^{3}}{n_{0}} \sqrt{\frac{1+\delta^{2}}{\tilde{h}_{m}^{3}+\delta^{2}}}, \\
B= & {\left[\frac{2}{\Delta z_{1}} \int_{\theta_{m_{1}}}^{\theta_{m_{2}}} h^{3} d \theta+\frac{z_{m_{2}}-z_{m_{1}}}{\Delta \theta_{1}}\left(h_{G}^{3}+h_{1}^{3}\right)\right.} \\
& \left.+\frac{2 \Lambda\left(z_{m_{2}}-z_{m_{1}}\right)}{p_{m}}\left(h_{G}-h_{1}\right)\right] p_{s}^{2} \tilde{h}_{m}^{2}, \\
C= & \frac{1}{\Delta z_{1}} \int_{\theta_{m_{1}}}^{\theta_{m_{2}}} h^{3} d \theta\left(Q_{j, i_{1}}^{(r-1)}+Q_{j, i_{2}}^{(r-1)}\right) \\
& +\frac{z_{m_{2}}-z_{m_{1}}}{\Delta \theta_{1}}\left(h_{G}^{3} Q_{j_{2}, i}^{(r-1)}+h_{1}^{3} Q_{j_{1}, i}^{(r-1)}\right), \quad r \geq 1 .
\end{aligned}
$$

The solution of (22) is obtained by using the chord method, because $x \in[0,1]$. In the case $x>1$, by using the transformation $x=1 / x^{\prime}$ and (22),

$$
A x^{\prime 2} G\left(x^{\prime}\right)-C x^{\prime 2}+B=0 .
$$

After (22) or (24) is solved, we can calculate $Q_{m}$ :

$$
Q_{m}= \begin{cases}\left(p_{s} x \tilde{h}_{m}\right)^{2}, & \text { if } x \leq 1, \\ \left(p_{s}\left(x^{\prime}\right)^{-1} \tilde{h}_{m}^{\prime}\right)^{2}, & \text { if } x>1 .\end{cases}
$$


In the inside points of the domain $\mathcal{D}$, the algebraic system of equations, which results by using the finite difference in (10), is

$$
\begin{aligned}
\frac{1}{\Delta z^{2}} & Q_{j, i-1}^{(r)}-2\left[\frac{1}{\Delta \theta^{2}}+\frac{1}{\Delta z^{2}}+\frac{1}{h_{j, i}}\left(\frac{\partial^{2} h}{\partial \theta^{2}}\right)_{j, i}\right] Q_{j, i}^{(r)}+\frac{1}{\Delta z^{2}} Q_{j, i+1}^{(r)} \\
& +\left\{\frac{1}{\Delta \theta^{2}}+\frac{1}{\Delta \theta h_{j, i}}\left[\left(\frac{\partial h}{\partial \theta}\right)_{j, i}+\frac{\Lambda}{\left(Q_{j, i}^{(r-1)}\right)^{1 / 2}}\right]\right\} Q_{j-1, i}^{(r)} \\
& +\left\{\frac{1}{\Delta \theta^{2}}-\frac{1}{\Delta \theta h_{j, i}}\left[\left(\frac{\partial h}{\partial \theta}\right)_{j, i}+\frac{\Lambda}{\left(Q_{j, i}^{(r-1)}\right)^{1 / 2}}\right]\right\} Q_{j+1, i}^{(r)} \\
= & 0
\end{aligned}
$$

where $j=\overline{1, N}, i=\overline{1, M-1}$.

For $j=1$ and $j=N$ in (26) is used the periodicity condition:

$$
Q_{0, i-1}^{(r)}=Q_{N, i-1}^{(r)}, \quad Q_{N+1, i}^{(r)}=Q_{1, i}^{(r)} .
$$

For $i=1$ and $i=M$ the conditions on the outside boundary become

$$
Q_{j, 1}^{(r)}=h_{j}^{2}, \quad Q_{j, M}^{(r)}=h_{j}^{2} .
$$

In the neighborhood of $C_{m}$ the equations are:

- if $(i-1) \cdot \Delta z \in\left[z_{m 1}, z_{m 2}\right]$ and $0<j \cdot \Delta \theta-\theta_{m 2}<\Delta \theta$, then

$$
\begin{aligned}
\frac{1}{\Delta z^{2}} Q_{j, i-1}^{(r)}-2\left\{\frac{1}{\Delta \theta \Delta \theta_{1}}+\frac{1}{\Delta z^{2}}+\frac{1}{2 h_{j, i}} \frac{\Delta \theta-\Delta \theta_{1}}{\Delta \theta \Delta \theta_{1}}\right. \\
\left.\times\left[\left(\frac{\partial h}{\partial \theta}\right)_{j, i}+\frac{\Lambda}{\left(Q_{j, i}^{(r-1)}\right)^{1 / 2}}\right]+\frac{1}{h_{j, i}}\left(\frac{\partial^{2} h}{\partial \theta^{2}}\right)_{j, i}\right\} Q_{j, i}^{(r)} \\
+\frac{1}{\Delta z^{2}} Q_{j, i-1}^{(r)}+\left\{\frac{2}{\Delta \theta\left(\Delta \theta+\Delta \theta_{1}\right)}+\frac{1}{h_{j, i}}\right. \\
\left.\times \frac{\Delta \theta_{1}}{\Delta \theta\left(\Delta \theta+\Delta \theta_{1}\right)}\left[\left(\frac{\partial h}{\partial \theta}\right)_{j, i}+\frac{\Lambda}{\left(Q_{j, i}^{(r-1)}\right)^{1 / 2}}\right]\right\} Q_{j-1, i}^{(r)} \\
=\left\{-\frac{2}{\Delta \theta\left(\Delta \theta+\Delta \theta_{1}\right)}+\frac{1}{h_{j, i}} \frac{\Delta \theta}{\Delta \theta_{1}\left(\Delta \theta+\Delta \theta_{1}\right)}\right. \\
\left.\times\left[\left(\frac{\partial h}{\partial \theta}\right)_{j, i}+\frac{\Lambda}{\left(Q_{j, i}^{(r-1)}\right)^{1 / 2}}\right]\right\} Q_{m}^{(r)} ;
\end{aligned}
$$

- if $(i-1) \cdot \Delta z \in\left[z_{m 1}, z_{m 2}\right]$ and $0<(j-1) \cdot \Delta \theta-\theta_{m 2}<\Delta \theta$, then

$$
\begin{aligned}
\frac{1}{\Delta z^{2}} Q_{j, i-1}^{(r)}-2\left\{\frac{1}{\Delta \theta \Delta \theta_{1}}+\frac{1}{\Delta z^{2}}+\frac{1}{2 h_{j, i}} \frac{\Delta \theta-\Delta \theta_{1}}{\Delta \theta \Delta \theta_{1}}\right. \\
\left.\times\left[\left(\frac{\partial h}{\partial \theta}\right)_{j, i}+\frac{\Lambda}{\left(Q_{j, i}^{(r-1)}\right)^{1 / 2}}\right]+\frac{1}{h_{j, i}}\left(\frac{\partial^{2} h}{\partial \theta^{2}}\right)_{j, i}\right\} Q_{j, i}^{(r)} \\
+\frac{1}{\Delta z^{2}} Q_{j, i-1}^{(r)}+\left\{\frac{2}{\Delta \theta\left(\Delta \theta+\Delta \theta_{1}\right)}-\frac{1}{h_{j, i}}\right.
\end{aligned}
$$




$$
\begin{aligned}
& \left.\times \frac{\Delta \theta_{1}}{\Delta \theta\left(\Delta \theta+\Delta \theta_{1}\right)}\left[\left(\frac{\partial h}{\partial \theta}\right)_{j, i}+\frac{\Lambda}{\left(Q_{j, i}^{(r-1)}\right)^{1 / 2}}\right]\right\} Q_{j+1, i}^{(r)} \\
= & \left\{-\frac{2}{\Delta \theta\left(\Delta \theta+\Delta \theta_{1}\right)}-\frac{1}{h_{j, i}} \frac{\Delta \theta}{\Delta \theta_{1}\left(\Delta \theta+\Delta \theta_{1}\right)}\left[\left(\frac{\partial h}{\partial \theta}\right)_{j, i}+\frac{\Lambda}{\left(Q_{j, i}^{(r-1)}\right)^{1 / 2}}\right]\right\} Q_{m}^{(r)} ;
\end{aligned}
$$

- if $(j-1) \cdot \Delta \theta \in\left[\theta_{m 1}, \theta_{m 2}\right]$ and $0<i \cdot \Delta z-z_{m 2}<\Delta z$, then

$$
\begin{aligned}
\frac{2}{\Delta z\left(\Delta z+\Delta z_{1}\right)} Q_{j, i+1}^{(r)}-2\left[\frac{1}{\Delta \theta^{2}}+\frac{1}{\Delta z \Delta z_{1}}+\frac{1}{h_{j, i}}\left(\frac{\partial^{2} h}{\partial \theta^{2}}\right)_{j, i}\right] Q_{j, i}^{(r)} \\
+\left\{\frac{1}{\Delta \theta^{2}}+\frac{1}{2 \Delta \theta h_{j, i}}\left[\left(\frac{\partial h}{\partial \theta}\right)_{j, i}+\frac{\Lambda}{\left(Q_{j, i}^{(r-1)}\right)^{1 / 2}}\right]\right\} Q_{j-1, i}^{(r)} \\
+\left\{\frac{1}{\Delta \theta^{2}}-\frac{1}{2 \Delta \theta h_{j, i}}\left[\left(\frac{\partial h}{\partial \theta}\right)_{j, i}+\frac{\Lambda}{\left(Q_{j, i}^{(r-1)}\right)^{1 / 2}}\right]\right\} Q_{j+1, i}^{(r)} \\
=-\frac{2}{\Delta z_{1}\left(\Delta z+\Delta z_{1}\right)} Q_{m}^{(r)} .
\end{aligned}
$$

The system of equations (26)-(31) can be written in a matrix form:

$$
\left[A_{j}\right]\left\{Q_{j}\right\}+\left[B_{j}\right]\left\{Q_{j-1}\right\}+\left[C_{j}\right]\left\{Q_{j+1}\right\}=\left\{R_{j}\right\}
$$

where $\left[A_{j}\right],\left[B_{j}\right],\left[C_{j}\right]$ are square matrices of dimension $M \times M$ and $\left\{Q_{j}\right\},\left\{Q_{j-1}\right\},\left\{Q_{j+1}\right\}$, $\left\{R_{j}\right\}$ are the column vectors of the unknown variable on the column $j, j-1, j+1$, and, respectively, the column vector of terms in the right-sides. Here the subscripts represent the iteration order. In (32) and in the relations that follow, we consider the iteration of order $r$.

In order to solve the system of equations (32) we use the method called 'column-wise'. This method is based on the relation which can be written for each $j$ :

$$
\left\{Q_{j-1}\right\}=\left[E_{j}\right]\left\{Q_{j}\right\}+\left\{F_{j}\right\}+\left[D_{j}\right]\left\{Q_{N}\right\} .
$$

Substituting (33) in (32), one finds, as a result,

$$
\left(\left[A_{j}\right]+\left[B_{j}\right]\left\{E_{j}\right\}\right)\left\{Q_{j}\right\}=-\left[C_{j}\right]\left\{Q_{j+1}\right\}+\left(\left\{R_{j}\right\}-\left[B_{j}\right]\left\{F_{j}\right\}\right)-\left[B_{j}\right]\left[D_{j}\right]\left\{Q_{N}\right\}
$$

By solving with regard to $\left\{Q_{j}\right\}$ and comparing with (33) we obtain the relations

$$
\begin{aligned}
& {\left[E_{j+1}\right]=-\left[T_{j}\right]\left[C_{j}\right],} \\
& {\left[F_{j+1}\right]=\left[T_{j}\right]\left(\left\{R_{j}\right\}-\left[B_{j}\right]\left\{F_{j}\right\}\right),} \\
& {\left[D_{j+1}\right]=-\left[T_{j}\right]\left[B_{j}\right]\left[D_{j}\right],}
\end{aligned}
$$

where

$$
\left[T_{j}\right]=\left(\left[A_{j}\right]+\left[B_{j}\right]\left[E_{j}\right]\right)^{-1} .
$$


The initial values for (35)-(38) are

$$
\left[E_{1}\right]=\left|\begin{array}{cccc}
0 & 0 & \ldots & 0 \\
0 & 0 & \ldots & 0 \\
. & . & \ldots & . \\
. & . & \ldots & . \\
. & . & \ldots & . \\
0 & 0 & \ldots & 0
\end{array}\right|, \quad\left\{F_{1}\right\}=\left|\begin{array}{c}
0 \\
0 \\
.
\end{array}\right| . \quad\left[D_{1}\right]=\left|\begin{array}{cccc}
1 & 0 & \ldots & 0 \\
0 & 1 & \ldots & 0 \\
. & . & \ldots & . \\
. & . & \ldots & . \\
. & . & \ldots & . \\
0 & 0 & \ldots & 1
\end{array}\right| .
$$

After the calculation of the matrix $\left[E_{j}\right],\left[D_{j}\right],\left\{F_{j}\right\}$, for $j=\overline{1, N}$, we write the relation for $j=N+1$ and use the periodicity condition $\left\{Q_{N+1}\right\}=\left\{Q_{1}\right\}$. We find, as a result,

$$
\left\{Q_{N}\right\}=\left(I-\left[D_{N+1}\right]\right)^{-1}\left(\left[E_{N+1}\right]\left\{Q_{1}\right\}+\left\{F_{N+1}\right\}\right) .
$$

From (33) and (40), written for $j=N, N-1, \ldots, 2$, we obtain the following relation between $\left\{Q_{j}\right\}$ and $\left\{Q_{1}\right\}$ :

$$
\left\{Q_{j}\right\}=\left[G_{j}\right]\left\{Q_{1}\right\}+\left\{S_{j}\right\}
$$

where

$$
\begin{aligned}
& {\left[G_{N}\right]=\left(I-\left[D_{N+1}\right]\right)^{-1}\left[E_{N+1}\right],} \\
& \left\{S_{N}\right\}=\left(I-\left[D_{N+1}\right]\right)^{-1}\left[F_{N+1}\right] .
\end{aligned}
$$

By means of (33) and (41) we obtain the relation for the calculation of $\left[G_{j}\right]$ and $\left\{S_{j}\right\}$, for $j=N, N-1, \ldots, 2$ :

$$
\begin{aligned}
& {\left[G_{j-1}\right]=\left[E_{j}\right]\left[G_{j}\right]+\left[D_{j}\right]\left[G_{N}\right],} \\
& \left\{S_{j-1}\right\}=\left[E_{j}\right]\left[S_{j}\right]+\left\{F_{j}\right\}+\left[D_{j}\right]\left\{S_{N}\right\} .
\end{aligned}
$$

After we compute the matrix $\left[G_{j}\right]$ and $\left\{S_{j}\right\}$, from (41), written for $j=1$, one finds, as a result,

$$
\left\{Q_{1}\right\}=\left(I-\left[G_{1}\right]\right)^{-1}\left\{S_{1}\right\}
$$

The other vectors $\left\{Q_{j}\right\}$ can be computed now from (41) for $j=\overline{2, N}$. The solution $Q^{(r)}$ is compared with $Q^{(r-1)}$. Our problem is solved if

$$
\frac{\sum_{j, i}\left|Q_{j, i}^{(r)}-Q_{j, i}^{(r-1)}\right|}{\sum_{j, i}\left|Q_{j, i}^{(r-1)}\right|}<\varepsilon
$$

where $\varepsilon$ is a positive number of a suitable choice.

If the last condition (47) is not valid, then the calculation is repeated for a new iteration $r+1$.

Figure 4 compares the attitude angle function of the bearing number for a compliant journal bearing at different eccentricity: 0.1, 0.4. Figure 5 represents the three dimensional plot of the hydrodynamic pressure distribution for the compliant journal bearing 
Figure 4 Numerical calculated attitude angles.

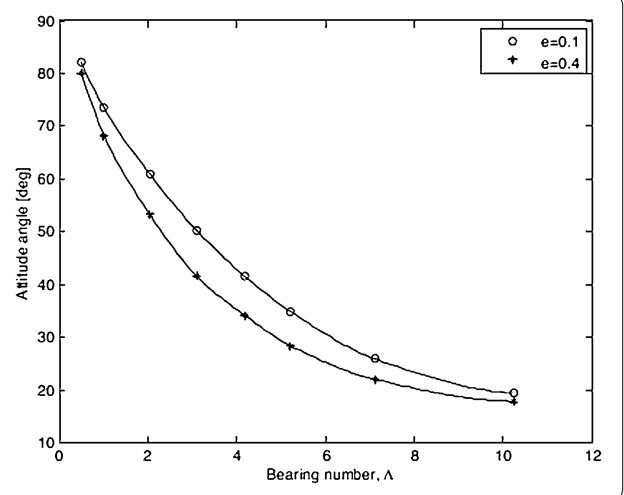

Figure 5 Numerical calculated pressure profiles.

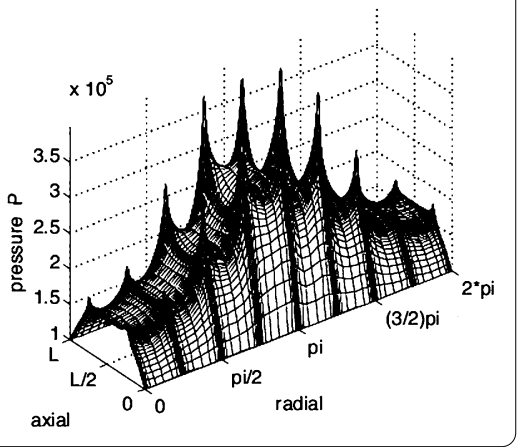

running at 30,000 rpm. The hydrodynamic pressure developed along the axial direction has a parabolic profile wherein the pressure is maximum at the mid-section or at the center of the bearing and the pressure is normalized at the ends of the bearing.

\section{Conclusions}

Even the boundary conditions become nonlinear integral-differential expressions with respect to the Reynolds equation, we can solve the problem by using the 'column-wise' technique.

Competing interests

The authors declare that they have no competing interests.

Authors' contributions

All authors contributed equally to the writing of this paper. All authors read and approved the final manuscript.

\section{Author details}

${ }^{1}$ Faculty of Mathematics and Computer Science, Transilvania University of Brasov, luliu Maniu Str., No. 50, Brasov, Romania. ${ }^{2}$ Department of Mathematics, Texas A \& M University - Kingsville, 700 University Blvd., Kingsville, TX 78363-8202, USA.

${ }^{3}$ Department of Mathematics, Faculty of Science, King Abdulaziz University, P.O. Box 80203, Jeddah, 21589, Saudi Arabia.

Received: 30 May 2015 Accepted: 14 October 2015 Published online: 29 October 2015

\section{References}

1. Truesdell, C, Rajagopal, KR: An Introduction in the Mechanics of Fluids. Birchauser, Boston (2009)

2. Castelli, V, Pirvics, J: Review of numerical methods in gas bearings film analysis. Technical Report, Columbia Univ., Research Lab. (2005)

3. Andres, LS: Turbulent flow, flexure-pivot hybrid bearings. J. Tribol. 118(1), 190-200 (1996)

4. Helene, M, Arhir, M, Frene, J: Specific aspects of hybrid bearings at high rotational speeds. Méc. Ind. 5, 651-658 (2004)

5. Ausman, JS: Gas-lubrification bearings. J. Basic Eng. 86, 328-336 (1965)

6. Nazurov, SA, Videman, JH: A modified nonlinear Reynolds equilibrium for thin viscous flows. Asymptot. Anal. 52(1-2), 1-36 (2007) 
7. Hellsten, A, Wallin, S: Explicit algebraic Reynolds stress and non-linear viscosity models. Int. J. Comput. Fluid Dyn. 23(4), 349-361 (2009)

8. Marin, M, Florea, O: On temporal behaviour of solutions in thermoelasticity of porous micropolar bodies. An. Ştiinţ. Univ. "Ovidius" Constanţa Ser. Mat. 22(1), 169-188 (2014)

9. Marin, M, Stan, G: Weak solutions in elasticity of dipolar bodies with stretch. Carpath. J. Math. 29(1), 33-40 (2013)

10. Marin, M, Agarwal, RP, Mahmoud, SR: Modeling a microstretch thermoelastic body with two temperatures. Abstr. Appl. Anal. 2013, Article ID 583464 (2013). doi:10.1155/2013/583464

11. Marin, M: On the minimum principle for dipolar materials with stretch. Nonlinear Anal., Real World Appl. 10, 1572-1578 (2009)

12. Fetecau, C, Prasad, SC, Rajagopal, KR: A note on the flow induced by a constantly accelerating plate in an Oldroyd-B fluid. Appl. Math. Model. 31, 647-654 (2007)

Submit your manuscript to a SpringerOpen ${ }^{\circ}$ journal and benefit from:

- Convenient online submission

- Rigorous peer review

- Immediate publication on acceptance

- Open access: articles freely available online

- High visibility within the field

Retaining the copyright to your article

Submit your next manuscript at $\boldsymbol{s p r i n g e r o p e n . c o m ~}$ 